\title{
Papillary adenocarcinoma of the stomach
}

\author{
Kazuhiro Yasuda, Yosuke Adachi, Norio Shiraishi, Seigo Maeo, and Seigo Kitano \\ First Department of Surgery, Oita Medical University, 1-1 Idaigaoka, Hasama-machi, Oita 879-5593, Japan
}

\begin{abstract}
Background. Papillary gastric carcinoma (PGC) is a rare histologic entity among gastric adenocarcinomas. The aim of this study was to clarify the clinicopathologic characteristics of PGC, including the survival rate, recurrence pattern, and factors influencing the prognosis of patients with PGC.

Methods. The clinicopathologic findings of 65 patients with PGC and 566 patients with non-papillary gastric carcinoma (NGC) were examined and compared. The tumor was classified as PGC when more than $50 \%$ of the tumor area contained papillary structures. Survival rates were calculated using the Kaplan-Meier method and were assessed by the generalized Wilcoxon test. Prognostic factors were evaluated by univariate analysis, using the $\chi^{2}$ test and Student's t-test, and confirmed by multivariate analysis, using the Cox proportional hazards model.

Results. PGC was characteristically found in patients over 65 years of age $(68 \%)$, was located in the upper one-third of the stomach $(37 \%)$, was of grossly localized type $(85 \%)$, was negative for serosal invasion $(86 \%)$, and was associated with liver metastasis (14\%). The overall 5-year survival rate for patients with PGC was significantly lower than the rate for those with NGC $(63 \%$ vs $76 \%)(P<0.05)$. Although the 5 year survival rate for each stage of PGC and NGC did not differ significantly, the death of PGC patients was more frequently associated with liver metastasis (62\%) than with peritoneal dissemination $(5 \%)$. Independent prognostic factors of PGC were liver metastasis, serosal invasion, and lymph node metastasis.

Conclusion. These results suggest that PGC is characterized by advanced patient age, proximal tumor location, grossly localized type, negative serosal invasion, and frequent liver metastasis. A poor prognosis for patients with PGC is associated with the presence or recurrence of liver metastasis.
\end{abstract}

Key words Stomach $\cdot$ Cancer-Gastric carcinoma-Papillary adenocarcinoma $\cdot$ Histologic type

Offprint requests to: $\mathrm{K}$. Yasuda

Received: January 11, 2000 / Accepted: April 7, 2000
Introduction

Gastric carcinoma has been divided into two major histologic types: well differentiated (intestinal, expanding, differentiated, and glandular) and poorly differentiated (diffuse, infiltrating, undifferentiated, and nonglandular) [1-4]. The former is characterized by expansive growth and hematogenous metastasis to the liver, and the latter by infiltrating growth and peritoneal dissemination. Among well differentiated gastric carcinomas, a tumor consisting of papillary structures with columnar epithelium is called a papillary adenocarcinoma [5]. This histologic subtype is rare, and its biological behavior and prognostic significance are unclear.

The aim of this study was to clarify the following: (1) the clinicopathologic features of papillary gastric carcinoma (PGC) compared with those of non-papillary gastric carcinoma (NGC); (2) the difference in 5-year survival rates and causes of tumor-related deaths in patients with PGC compared with patients with NGC; and (3) the clinicopathologic factors influencing the prognosis of patients with PGC.

\section{Patients and methods}

During the period from January 1982 to July 1998, 631 patients with gastric adenocarcinoma, but no evidence of any other malignancy, underwent gastrectomy at the First Department of Surgery, Oita Medical University. Of the 631 patients, PGC was demonstrated in 65 $(10.3 \%)$. PGC is composed of papillary epithelial processes with thin fibrous cores (Fig. 1). We defined PGC as a tumor in which more than $50 \%$ of the tumor area contained papillary structures. Of the 65 PGCs, 27 were early gastric carcinomas, and 38 were advanced gastric carcinomas. A tumor was defined as early gastric carcinoma when the invasion was limited to the mucosa 


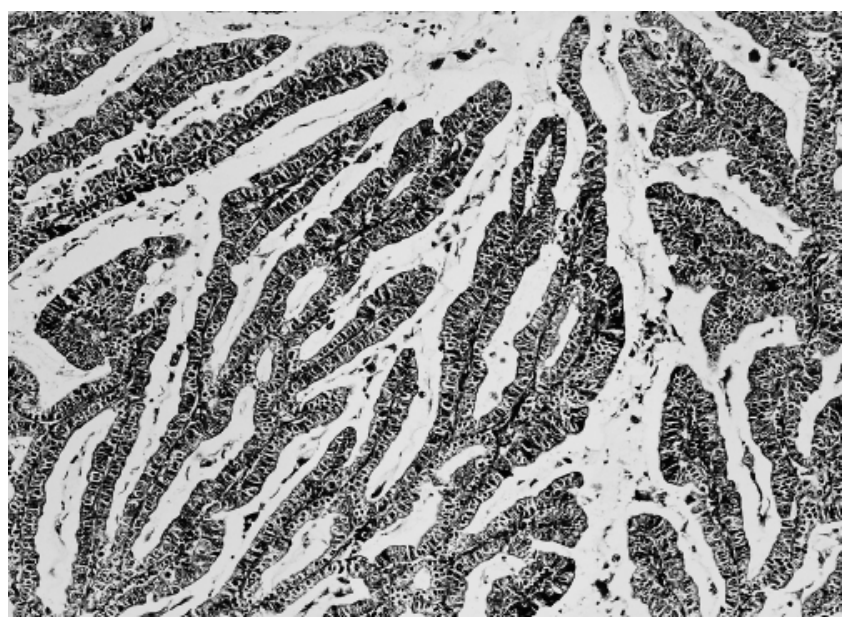

Fig. 1. Papillary adenocarcinoma of the stomach. H\&E, $\times 25$

or submucosa, and as advanced gastric carcinoma when it invaded the muscularis propria or deeper, regardless of the presence or absence of lymph node metastasis [5].

The age and sex of the patient, type of operation, location of the tumors, and presence or absence of liver metastasis and peritoneal dissemination were determined from medical charts. Gross appearance was examined using photographs of the resected specimens, and tumor size was given as the maximal diameter. All specimens were fixed in a $10 \%$ formalin solution and cut along the largest diameter of the tumor or along its deepest invasion. Four- $\mu \mathrm{m}$-thick sections were cut from paraffin-embedded tissues, stained with hematoxylin and eosin $(\mathrm{H} \& \mathrm{E})$, and examined to obtain histologic findings such as depth of invasion, lymphatic and vascular permeations, and status of lymph node metastasis. The stage of the disease and operative curability were estimated, and the patients' outcomes and causes of death were examined. These clinicopathologic findings were investigated according to the Japanese classification of gastric carcinoma outlined by the Japanese Gastric Cancer Association [5]. Serosal invasion was defined as tumor involvement of the serosal surface or of the adjacent organs. Surgery was regarded as curative when the macroscopic tumor tissue was completely removed during surgery and histology showed the resection margin to be free of tumor cells.

Differences in clinicopathologic data between PGC and NGC were analyzed by the $\chi^{2}$ test and Student's $t$ test. Survival rates were calculated using the KaplanMeier method and were assessed by the generalized Wilcoxon test. Multivariate analysis, by the Cox proportional hazards model, was performed to determine independent prognostic factors. A $P$ value of less than 0.05 was regarded as statistically significant.

\section{Results}

Significant differences between PGC and NGC were seen in the frequency of patients over 65 years of age ( $68 \%$ vs $51 \%$ ), tumor location in the upper one-third of the stomach ( $37 \%$ vs $19 \%$ ), grossly localized type $(85 \%$ vs $67 \%)$, negative serosal invasion $(86 \%$ vs $73 \%)$, and occurrence of liver metastasis (14\% vs $3 \%)$ (Table 1$)$. Advanced PGC, compared with advanced NGC, was characteristically located in the upper one-third of the stomach $(50 \%$ vs $25 \%)$ and grossly localized $(74 \%$ vs $38 \%$ ), with negative serosal invasion ( $76 \%$ vs $49 \%)$ and frequent liver metastasis (24\% vs $5 \%$ ). Early PGC, compared with early NGC, was frequently seen in patients over 65 years of age $(74 \%$ vs $50 \%)$ and was the grossly elevated type (67\% vs $21 \%)$. When 65 patients with PGC were compared with age- and sex-matched patients with NGC, the difference between the two groups was also significant in the tumor location and liver metastasis (Table 2).

The overall 5-year survival rate was significantly lower for patients with PGC than for patients with NGC (63\% vs 76\%) (Fig. 2). When the same stage of disease was compared, however, the 5-year survival rates were not different between PGC and NGC (Fig. 3). Of 65 patients with PGC, 21 (32\%) died of recurrence. Of these 21 patients, liver metastasis occurred in $13(62 \%)$ and peritoneal dissemination in $1(5 \%)$.

Clinicopathologic factors influencing the prognosis of patients with PGC were the type of gastrectomy, tumor size, gross appearance, serosal invasion, lymphatic and vascular permeations, lymph node metastasis, peritoneal dissemination, liver metastasis, stage of disease, and operative curability (Table 3). Of these 11 factors, liver metastasis, serosal invasion, and lymph node metastasis independently affected the prognosis of patients with PGC (Table 4).

\section{Discussion}

PGC is a rare histologic type among adenocarcinomas of the stomach. Recent Japanese reports showed that PGC accounted for only $6 \%$ to $11 \%$ of gastric carcinomas [6,7], and the incidence in our series $(10 \%)$ was in accordance with these data. In this study, we clarified the clinicopathologic characteristics of PGC, which include occurrence in aged patients, proximal tumor location, gross appearance of an elevated type in the early stage and a localized type in the advanced stage, negative serosal invasion, frequent liver metastasis, and, ultimately, poor patient outcomes.

Nakamura et al. [8] investigated the clinicopathologic findings of 7031 patients with gastric carcinoma and found that the histologic type did not influence the 
Table 1. Clinicopathologic data for papillary and non-papillary gastric carcinomas

\begin{tabular}{|c|c|c|c|}
\hline & $\begin{array}{c}\text { PGC } \\
(n=65)\end{array}$ & $\begin{array}{c}\text { NGC } \\
(n=566)\end{array}$ & $P$ value \\
\hline Age (years) & $67.7 \pm 10.6$ & $63.0 \pm 12.0$ & $<0.01$ \\
\hline Male sex & $49(75 \%)$ & $385(68 \%)$ & NS \\
\hline Total gastrectomy & $23(35 \%)$ & $177(31 \%)$ & NS \\
\hline Location in the upper one-third & $24(37 \%)$ & $106(19 \%)$ & $<0.01$ \\
\hline Size $(\mathrm{cm})$ & $5.2 \pm 3.0$ & $5.3 \pm 3.9$ & NS \\
\hline Grossly localized type & $55(85 \%)$ & $379(67 \%)$ & $<0.01$ \\
\hline \multicolumn{4}{|l|}{ Serosal invasion } \\
\hline Present & $9(14 \%)$ & $154(27 \%)$ & $<0.05$ \\
\hline \multicolumn{4}{|l|}{ Lymphatic permeation } \\
\hline Present & $19(29 \%)$ & $168(30 \%)$ & NS \\
\hline \multicolumn{4}{|l|}{ Venous permeation } \\
\hline Present & $5(8 \%)$ & $22(4 \%)$ & NS \\
\hline \multicolumn{4}{|l|}{ Lymph node metastasis } \\
\hline Present & $35(54 \%)$ & $246(43 \%)$ & NS \\
\hline \multicolumn{4}{|l|}{ Peritoneal dissemination } \\
\hline Present & $3(5 \%)$ & $29(5 \%)$ & NS \\
\hline \multicolumn{4}{|l|}{ Liver metastasis } \\
\hline Present & $9(14 \%)$ & $17(3 \%)$ & $<0.01$ \\
\hline Stage III, IV & $24(37 \%)$ & $217(38 \%)$ & NS \\
\hline Curative operation & $56(86 \%)$ & $504(89 \%)$ & NS \\
\hline
\end{tabular}

Values for age and size are expressed as means \pm SD

PGC, Papillary gastric carcinoma; NGC, non-papillary gastric carcinoma; NS, not significant

Table 2. Clinicopathologic data for papillary and age- and sex-matched non-papillary gastric carcinomas

\begin{tabular}{|c|c|c|c|}
\hline & $\begin{array}{c}\text { PGC } \\
(n=65)\end{array}$ & $\begin{array}{l}\text { Age- and sex-matched } \\
\text { NGC }(n=65)\end{array}$ & $P$ value \\
\hline Total gastrectomy & $23(35 \%)$ & $16(25 \%)$ & NS \\
\hline Location in the upper one-third & $24(37 \%)$ & $12(18 \%)$ & $<0.05$ \\
\hline Size $(\mathrm{cm})$ & $5.2 \pm 3.0$ & $4.5 \pm 2.8$ & NS \\
\hline Grossly localized type & $55(85 \%)$ & $46(71 \%)$ & NS \\
\hline \multicolumn{4}{|l|}{ Serosal invasion } \\
\hline Present & $9(14 \%)$ & $17(27 \%)$ & NS \\
\hline \multicolumn{4}{|l|}{ Lymphatic permeation } \\
\hline $\begin{array}{l}\text { Present } \\
\text { Venous permeation }\end{array}$ & $19(29 \%)$ & $17(27 \%)$ & NS \\
\hline Present & $5(8 \%)$ & $2(3 \%)$ & NS \\
\hline \multicolumn{4}{|l|}{ Lymph node metastasis } \\
\hline Present & $35(54 \%)$ & $26(40 \%)$ & NS \\
\hline $\begin{array}{l}\text { Peritoneal dissemination } \\
\text { Present }\end{array}$ & $3(5 \%)$ & $2(3 \%)$ & NS \\
\hline \multicolumn{4}{|l|}{ Liver metastasis } \\
\hline Present & $9(14 \%)$ & $2(3 \%)$ & $<0.05$ \\
\hline Stage III, IV & $24(37 \%)$ & $20(31 \%)$ & NS \\
\hline Curative operation & $56(86 \%)$ & $61(94 \%)$ & NS \\
\hline
\end{tabular}

Values for size are expressed as means \pm SD

PGC, Papillary gastric carcinoma; NGC, non-papillary gastric carcinoma; NS, not significant

prognosis after gastrectomy, except in special types such as adenosquamous and squamous cell carcinomas $[9,10]$. In their study, however, papillary adenocarcinoma was classified as well differentiated adenocarcinoma, and the prognostic value of PGC was not evaluated. Maruyama [11] undertook a multivariate analysis of 3994 patients who had gastric carcinoma without distant metastasis, and demonstrated that histologic type was one of five prognostic factors independently influencing the survival of patients.

In a Japanese report, Hirota et al. [7] indicated that when tumors were classified by the depth of wall 
invasion, papillary adenocarcinoma was associated with frequent lymph node metastasis and a low survival rate. They divided 6276 gastric carcinomas into six histologic types: papillary adenocarcinoma $(n=699)$, well differentiated tubular adenocarcinoma $(n=1081)$, moderately differentiated tubular adenocarcinoma $(n$ $=1159)$, poorly differentiated adenocarcinoma $(n=$ $1628)$, mucinous adenocarcinoma $(n=228)$, and signetring cell carcinoma $(n=1481)$. The frequency of lymph node metastasis was highest in papillary adenocarcinoma, occuring in $32 \%$ of tumors invading the submucosa, $56 \%$ of those invading the muscularis propria, and $75 \%$ of those invading the subserosa, when compared with the frequencies of lymph node

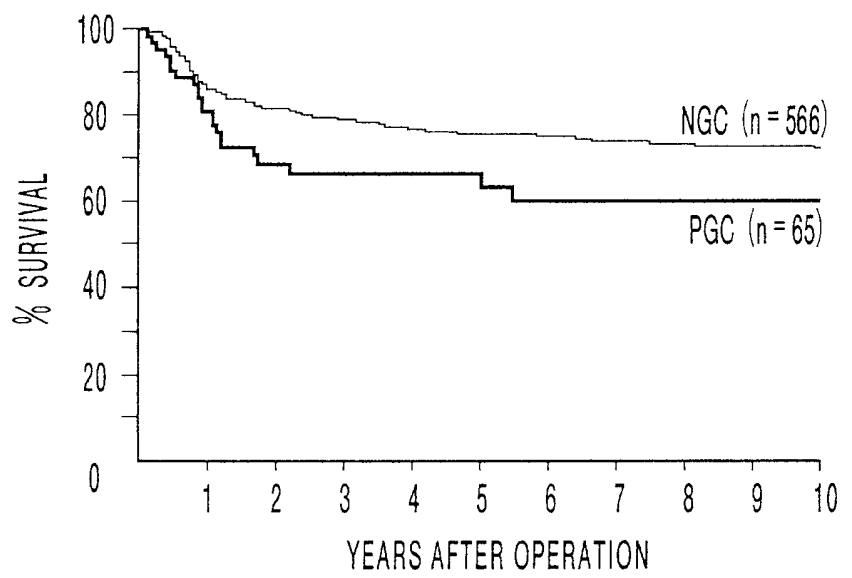

Fig. 2. Survival curves for 65 patients with papillary gastric carcinoma $(P G C)$ and 566 patients with non-papillary gastric carcinoma $(N G C)$ metastasis in all tumors of $18 \%, 48 \%$, and $62 \%$, respectively. The 10 -year survival rates in patients with papillary adenocarcinomas that invaded the submucosa, muscularis propria, and subserosa were $61 \%, 61 \%$, and $33 \%$, respectively, while the 10 -year survival rates for all tumors were $76 \%, 70 \%$, and $51 \%$, respectively. These differences were significant. Hirota and colleagues [7] therefore concluded that, except for tumors limited to the mucosa and those invading down to the serosal surface, papillary adenocarcinoma was significantly correlated with a poor prognosis, because of its frequent lymph node metastasis.

In our study, the difference in the frequency of lymph node metastasis between PGC (54\%) and NGC (43\%) was not significant. When mucosal cancers and cancers with serosal invasion were excluded, the frequency of lymph node metastasis was $65 \%$ in PGC $(n=40)$ and $40 \%$ in NGC $(n=265)$, which was a significant difference $(P<0.01)$. Our data, therefore, agree with the findings reported by Hirota and colleagues [7]. However, the 5-year survival rate of our patients with PGC (63\%) was higher than that of their patients with PGC (47\%). The frequency of serosal invasion in our series $(14 \%)$ was significantly lower than that in their patients $(40 \%)(P<0.01)$. We consider that this difference in the frequency of serosal invasion correlated with the difference between the 5-year survival rate in our study and that reported by Hirota et al. [7].

Liver metastasis and peritoneal dissemination are the most serious progressions of gastric carcinoma [12]. It is well documented that the former is most frequently
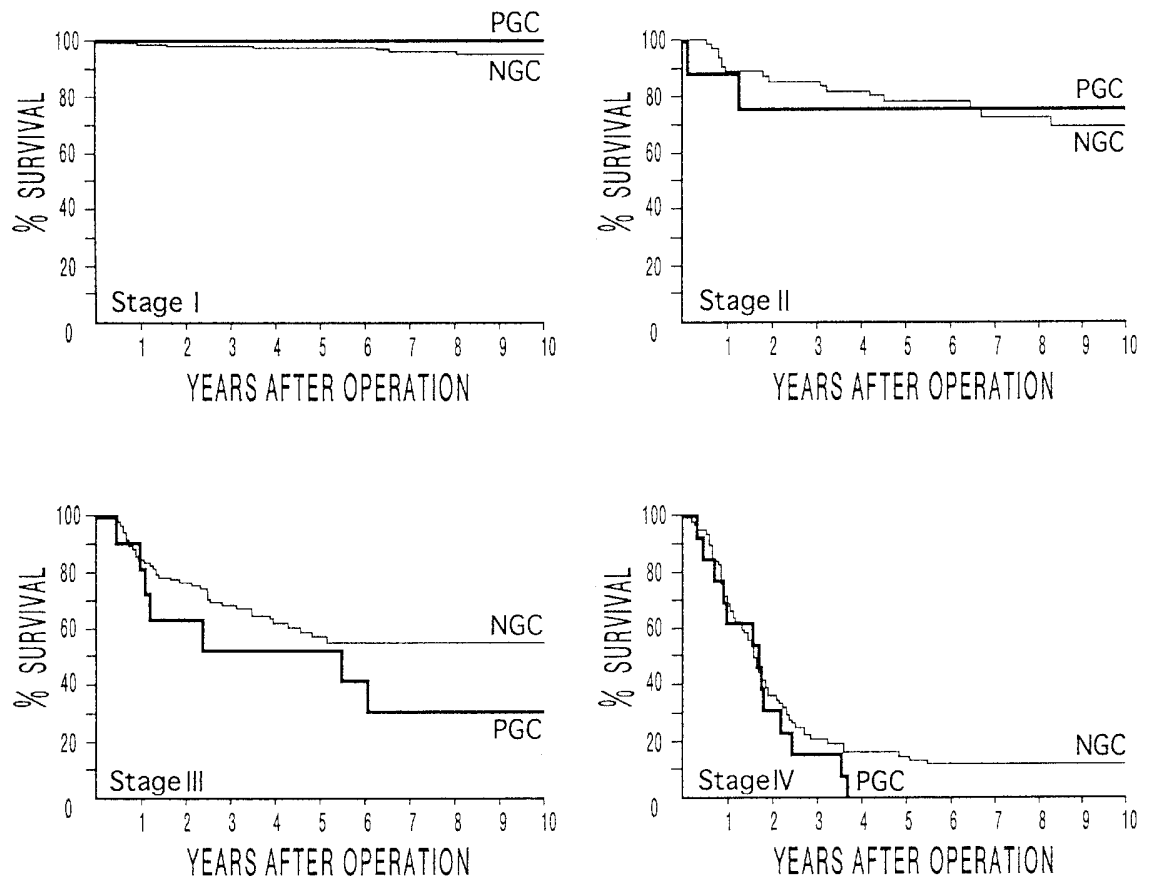

Fig. 3. Survival curves for patients with papillary gastric carcinoma $(P G C)$ and non-papillary gastric carcinoma $(N G C)$, according to the stage of disease 
Table 3. Factors influencing the prognosis of patients with papillary gastric carcinoma

\begin{tabular}{|c|c|c|c|}
\hline & No. of patients & 5-Year survival rate $(\%)$ & $P$ value \\
\hline \multicolumn{4}{|l|}{ Age (years) } \\
\hline$\leq 65$ & 23 & 61 & \\
\hline$>65$ & 42 & 63 & NS \\
\hline \multicolumn{4}{|l|}{ Sex } \\
\hline Male & 49 & 65 & \\
\hline Female & 16 & 59 & NS \\
\hline \multicolumn{4}{|l|}{ Gastrectomy } \\
\hline Distal, proximal & 42 & 80 & \\
\hline Total & 23 & 36 & $<0.01$ \\
\hline \multicolumn{4}{|l|}{ Location } \\
\hline Lower two-thirds & 41 & 74 & \\
\hline Upper one-third & 24 & 46 & NS \\
\hline \multicolumn{4}{|l|}{ Tumor size $(\mathrm{cm})$} \\
\hline$\leq 4$ & 29 & 93 & \\
\hline$>4$ & 36 & 40 & $<0.01$ \\
\hline \multicolumn{4}{|l|}{ Gross appearance } \\
\hline Localized & 55 & 68 & \\
\hline Infiltrative & 10 & 40 & $<0.05$ \\
\hline \multicolumn{4}{|l|}{ Serosal invasion } \\
\hline Absent & 56 & 77 & \\
\hline Present & 9 & 0 & $<0.01$ \\
\hline \multicolumn{4}{|c|}{ Lymphatic permeation } \\
\hline Absent & 46 & 78 & \\
\hline Present & 19 & 32 & $<0.01$ \\
\hline \multicolumn{4}{|l|}{ Vascular permeation } \\
\hline Absent & 60 & 67 & \\
\hline Present & 5 & 0 & $<0.01$ \\
\hline \multicolumn{4}{|c|}{ Lymph node metastasis } \\
\hline Absent & 30 & 100 & \\
\hline Present & 35 & 34 & $<0.01$ \\
\hline \multicolumn{4}{|c|}{ Peritoneal dissemination } \\
\hline Absent & 62 & 67 & \\
\hline Present & 3 & 0 & $<0.01$ \\
\hline \multicolumn{4}{|l|}{ Liver metastasis } \\
\hline Absent & 56 & 74 & \\
\hline Present & 9 & 0 & $<0.01$ \\
\hline \multicolumn{4}{|l|}{ Stage of disease } \\
\hline I, II & 41 & 95 & \\
\hline III, IV & 24 & 19 & $<0.01$ \\
\hline \multicolumn{4}{|l|}{ Operative curability } \\
\hline Curative & 56 & 76 & \\
\hline Non-curative & 9 & 0 & $<0.01$ \\
\hline
\end{tabular}

NS, not significant

Table 4. Independent prognostic factors in patients with papillary gastric carcinoma

\begin{tabular}{lccc}
\hline Variable & $\begin{array}{c}\text { Coefficient } \\
\text { of variation }\end{array}$ & $\chi^{2}$ & $P$ value \\
\hline Liver metastasis & 2.1534 & 7.2391 & 0.0071 \\
Serosal invasion & 1.3429 & 6.8569 & 0.0088 \\
Lymph node metastasis & 1.0213 & 4.9602 & 0.0259 \\
\hline
\end{tabular}

found in histologically well differentiated tumors, while the latter is often seen in poorly differentiated tumors [1-4,13]. Kaibara et al. [14] examined 71 patients with gastric carcinoma associated with synchronous or metachronous metastasis to the liver and showed that papillary adenocarcinoma was the most frequent histologic type (37\%), followed by poorly differentiated adenocarcinoma with a medullary growth pattern (31\%). They suggested that, as most papillary adenocarcinomas exhibited a medullary growth pattern, metastasis to the liver from gastric carcinoma was significantly associated with a medullary growth pattern, irrespective of the histologic type.

We previously investigated 74 patients with poorly differentiated medullary carcinoma of the stomach and showed that they were characterized by simultaneous or recurrent metastasis to the liver [15]. Because papillotubular differentiation in the mucosa of the tumor margin was seen in 28 tumors $(38 \%)$, it was 
concluded that some of the poorly differentiated medullary carcinomas arose as papillary adenocarcinomas in the gastric mucosa.

Our study also clarified the clinicopathologic factors influencing the prognosis of patients with PGC. On the univariate analysis, a poor outcome was associated with large tumor size, grossly infiltrative growth, and the presence of serosal invasion, lymphatic and vascular permeations, lymph node metastasis, peritoneal dissemination, and liver metastasis. However, multivariate analysis revealed that the most important prognostic factor was liver metastasis, followed by serosal invasion and lymph node metastasis. To improve the surgical outcome for PGC, preoperative and postoperative examinations for liver metastasis are important. When the histology of a biopsy specimen shows PGC, liver metastasis must be checked by ultrasonography and computed tomography prior to surgery [16,17]. Even if liver metastasis is not detected and curative gastrectomy is carried out, we must remember that, in patients with PGC, there may be later recurrence in the liver, resulting in tumor-related death.

In conclusion, although $\mathrm{PGC}$ is a rare histologic entity among gastric carcinomas, its biologic behavior is characterized by occurrence in aged patients, proximal tumor location, frequent liver metastasis, and poor surgical outcome even after curative gastrectomy.

\section{References}

1. Lauren P. The two histological main types of gastric carcinoma: diffuse and so-called intestinal-type carcinoma. Acta Pathol Microbiol Scand 1965;64:31-49.

2. Ming SC. Gastric carcinoma: a pathobiological classification. Cancer 1977;39:2475-85.

3. Sugano H, Nakamura K, Kato Y. Pathological studies of human gastric cancer. Acta Pathol Jpn 1982;32 (Suppl 2):329-47.
4. Esaki Y, Hirayama R, Hirokawa K. A comparison of patterns of metastasis in gastric cancer by histologic type and age. Cancer 1990;65:2086-90.

5. Japanese Gastric Cancer Association. Japanese classification of gastric carcinoma: 2nd English ed. Gastric Cancer 1998;1:10 24.

6. Uefuji K, Ichikura T, Tamakuma S. Clinical and prognostic characteristics of papillary clear carcinoma of stomach. Surg Today 1996;26:158-63.

7. Hirota T, Ochiai A, Itabashi M, Maruyama K. Significance of histological type of gastric carcinoma as a prognostic factor (in Japanese with English abstract). Stomach and Intestine 1991; 26:1149-58.

8. Nakamura K, Ueyama T, Yao T, Xuan ZX, Ambe K, Adachi Y, et al. Pathology and prognosis of gastric carcinoma: findings of 10000 patients who underwent primary gastrectomy. Cancer 1992;70:1030-7.

9. Mori M, Iwashita A, Enjoji M. Adenosquamous carcinoma of the stomach: a clinicopathologic analysis of 28 cases. Cancer 1986;57:333-9.

10. Mori M, Iwashita A, Enjoji M. Squamous cell carcinoma of the stomach: report of three cases. Am J Gastroenterol 1986;81:33942.

11. Maruyama K. The most important prognostic factors for gastric cancer patients. Scand J Gastroenterol 1987;22 (Suppl 133):63-8.

12. Maehara Y, Moriguchi S, Kakeji Y, Kohnoe S, Korenaga D, Haraguchi M, et al. Pertinent risk factors and gastric carcinoma with synchronous peritoneal dissemination or liver metastasis. Surgery 1991;110:820-3.

13. Adachi Y, Mori M, Enjoji M, Sugimachi K. Microvascular architecture of early gastric carcinoma: microvascularhistopathologic correlates. Cancer 1993;72:32-6.

14. Kaibara N, Kimura O, Nishidoi H, Makino M, Kawasumi H, Koga S. High incidence of liver metastasis in gastric cancer with medullary growth pattern. J Surg Oncol 1985;28:195-8.

15. Adachi Y, Mori M, Maehara Y, Sugimachi K. Poorly differentiated medullary carcinoma of the stomach. Cancer 1992;70:14626.

16. Cho JS, Kim JK, Rho SM, Lee HY, Jeong HY, Lee CS. Preoperative assessment of gastric carcinoma: value of two-phase dynamic CT with mechanical IV injection of contrast material. Am J Roentgenol 1994;163:69-75.

17. Adachi Y, Sakino I, Matsumata T, Iso Y, Yoh R, Kitano S, et al. Preoperative assessment of advanced gastric carcinoma using computed tomography. Am J Gastroenterol 1997;92:8725 . 